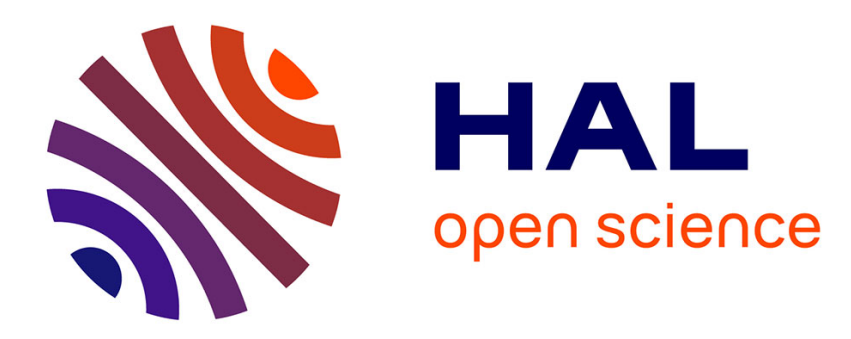

\title{
Intellektuelle Öffentlichkeit. Friedrich von Raumers Weg zwischen Politik und Wissenschaft
}

\author{
Anne Baillot
}

\section{To cite this version:}

Anne Baillot. Intellektuelle Öffentlichkeit. Friedrich von Raumers Weg zwischen Politik und Wissenschaft. Roland Berbig; Iwan d'Aprile; Helmut Peitsch; Erhard Schütz. Berlins 19. Jahrhundert. Ein Metropolen-Kompendium, p 135-146, 2011, 978-3-05-005083-6. hal-02455761

\section{HAL Id: hal-02455761 \\ https://hal.science/hal-02455761}

Submitted on 26 Jan 2020

HAL is a multi-disciplinary open access archive for the deposit and dissemination of scientific research documents, whether they are published or not. The documents may come from teaching and research institutions in France or abroad, or from public or private research centers.
L'archive ouverte pluridisciplinaire HAL, est destinée au dépôt et à la diffusion de documents scientifiques de niveau recherche, publiés ou non, émanant des établissements d'enseignement et de recherche français ou étrangers, des laboratoires publics ou privés. 


\section{Intellektuelle Öffentlichkeit. Friedrich von Raumers Weg zwischen Politik und Wissenschaft}

\section{Anne Baillot}

Mit einem außergewöhnlich langen Leben (1781-1873), einem umfangreichen Werk und einem strategischen Wirken im preußischen Staatsdienst scheinen in der Biographie Friedrich von Raumers alle Bedingungen zu einer intensiven Rezeption versammelt zu sein. Dennoch wollte es die Geschichte anders, und weder mit dem Werk noch mit dem Mensch setzt sich die heutige Forschung grundlegend auseinander. Seit Raumers Tod ist die Sekundärliteratur zu seinem Wirken und Schreiben eher spärlich. ${ }^{1}$ Dabei bieten sein veröffentlichter und sein unveröffentlichter Nachlass einen vielschichtigen Einblick in die Intellektuellennetzwerke des 19. Jahrhunderts. Genauer gesagt: Friedrich von Raumer gehört zu den Persönlichkeiten, bei denen der Begriff des Intellektuellen - an der Schnittstelle zwischen wissenschaftlicher und politischer Tätigkeit - besonders zutreffend ist. Ein Hinweis auf die essentielle Doppelspurigkeit seines Lebenswegs wurde bereits von Werner Friedrich seiner Dissertation Friedrich von Raumer als Historiker und Politiker programmatisch vorangestellt. So beschränkt sich die Einleitung der bislang einzigen Raumer-Mongraphie auf folgende Sätze:

Eine Würdigung Friedrich von Raumers, die nur seine wissenschaftlich-gelehrte Tätigkeit berücksichtigt, trifft nicht das Ganze, Wesentliche seiner Persönlichkeit. Raumer wollte mehr sein als ein Gelehrter und ist es auch gewesen. Er war Historiker, Staatslehrer, Politiker und Weltreisender. Es entsprach seiner Auffassung vom Wesen eines echten Historikers, sowie seiner vielseitigen, beweglichen Natur, wenn er allen diesen Gebieten sein Interesse zuwandte. Insbesondere ist für Raumer charakteristisch die enge Verbindung von historisch-gelehrter und praktisch-politischer Arbeit. Gerade die lebendige Wechselwirkung, in der beide Tätigkeiten bei ihm stehen, machen die Eigentümlichkeit seines Lebenswerkes aus. Eine Darstellung, die seiner Persönlichkeit gerecht werden will, muß besonders diesen Punkt berücksichtigen. ${ }^{2}$

Diese empathische Schilderung weist bei aller subjektiven Zuspitzung auf ein zentrales Element hin. So geht es auch in den folgenden Seiten darum, die Wechselbeziehungen zwischen gelehrten und politischen Ansprüchen in Raumers Lebensweg zu beleuchten.

Der 1781 Geborene kann eine ungewöhnliche Karriere vorweisen. Zwar kam er nicht als Unbekannter um 1800 nach Berlin, denn die Raumers waren bereits im höheren Staatsdienst aktiv und darüber hinaus mit der ebenfalls gut positionierten Familie von Gerlach verwandt. Dennoch verdankte er es bei weitem nicht nur seinen adligen Vorfahren und verwandten Beamten, dass ihn Hardenberg 1810 zu seinem engsten Mitarbeiter machte. ${ }^{3}$ In dieser Funktion hatte er als kaum Dreißigjährigen einen aktiven Anteil am großen politischen Reformwerk Preußens, von den schlesischen Agrarreformen bis hin zu dem Streit um die

\footnotetext{
${ }^{1} \mathrm{Zu}$ Leben und Werk Friedrich von Raumers ist immer noch auf die Dissertation Werner Friedrichs hinzuweisen: Friedrich von Raumer als Historiker und Politiker, Leipzig 1929. Doch sowohl W. Friedrich als auch der Historiograph Herman von Raumer in: Die Geschichte der Familie von Raumer, Neustadt/Aisch 1975 basieren hauptsächlich auf von Raumers eigenen Aussagen aus seinen Lebenserinnerungen und Briefwechsel (2 Bde, Leipzig 1861) bzw. aus seinem Literarischen Nachlaß (2 Bde, Berlin 1869).

${ }^{2}$ W. Friedrich (wie Anm. 1), S. 9. Daraufhin werden allerdings beide Aspekte getrennt untersucht: Kap.1 Bildung, Kap. 2 Zeit bei Hardenberg; Kap. 3 und 4 wissenschaftliche Tätigkeit; Kap. 5 politische Tätigkeit; Kap. 6 ,geistesgeschichtliche Stellung“.

${ }^{3} \mathrm{Zu}$ Hardenbergs Motiven, s. W. Friedrich (wie Anm. 1), S. 15 und H. von Raumer (wie Anm. 1), S. 102.
} 
Berliner Abendblätter, wo er eine entscheidende Rolle spielte. ${ }^{4} 1811$ verabschiedete er sich aus der Politik und wandte sich einer akademischen Laufbahn zu. Er erhielt zunächst einen Ruf als Staatswissenschaftler nach Breslau, wo er jedoch nach kurzer Zeit schon sein bevorzugtes Fach lehren konnte: die Geschichtswissenschaft. 1819 bekam er einen Ruf nach Berlin, wo er bis in seinen hohen Jahren hinein Vorlesungen über die Historie hielt. Im Laufe seiner Berliner Jahre wurde er auch Mitglied der preußischen Akademie der Wissenschaften. Seine meist bekannten Werke sind die Geschichte der Hohenstaufen ${ }^{5}$ und das Historische Taschenbuch $^{6}$. Darüber hinaus veröffentlichte er zahlreiche andere Schriften, die einen mehr historisch-politischer, die anderen philologischer, ${ }^{7}$ oder gar belletristischer Natur. ${ }^{8}$ Die Berichte, die er $\mathrm{zu}$ seinen zahlreichen Reisen ${ }^{9}$ verfasste, trugen $\mathrm{zu}$ seinem nationalen und internationalen Ruhm bei.

1. In den folgenden Abschnitten möchte ich kurz drei entscheidende Episoden aus dem Leben Friedrich von Raumers vorstellen, in denen sich das Gleichgewicht zwischen gelehrtem Beruf und politischen Überzeugungen schwer zu halten erwies: die Anpassung an das akademische Milieu als Professor in Breslau (1), das Amt am Oberzensurkollegium, das er gegen seine Überzeugung bezog und aufgrund seiner Überzeugung verließ (2) und schließlich das Amt als AkademieMitglied, das er ebenfalls nicht zufällig aufgeben musste (3). Diese Etappen aus seiner langen Laufbahn werden anhand von z.T. noch unedierten Briefen ${ }^{10}$ beleuchtet.Vom ,kleinen Staatskanzler611 zum Breslauer Professor

Selbst für die damalige gelehrte Welt gehört Raumers Abwendung von der Politik $1811 \mathrm{zu}$ den prägnantesten Merkmalen seiner Persönlichkeit. Noch bei der Aufnahme Raumers an der Akademie der Wissenschaften 1827, lange Zeit nachdem seine schließlich doch kurze politische Karriere ein Ende genommen hatte, kam Schleiermacher in seiner Begrüßungsrede emphatisch darauf zurück:

Ihre Leistungen liegen der Welt und uns vor Augen, und eine sichrere Bürgschaft dafür, daß ein Mann sein Leben ausschließend der Wissenschaft widmen wolle, giebt es nicht, als welche Sie geleistet haben, indem Sie eine politische Laufbahn voll der glänzendsten Aussichten verließen, aber in einem solchen Sinne und unter solchen

\footnotetext{
${ }^{4}$ Dazu, s. Lebenserinnerungen (wie Anm. 1), Bd. 1, S. 227-237; W. Friedrich (wie Anm. 1), S. 15-17. Der Streit um die Abendblätter besiegelt für Werner Friedrich den Bruch mit den romantischen Jugendfreunden: „Als Raumer [...] unter Hardenberg arbeitete und Muller und Arnim sich der Opposition gegen den Staatskanzler anschlossen, wurden die Freunde zu politischen Feinden. Raumer hat dann beim Niederschreiben seiner Biographie diese Jugendfreundschaft zu verschweigen gesucht." (S. 12) W. Friedrich hat bestimmt solche Passagen wie Lebenerinnerungen (wie Anm. 1), S. 260 im Blick.

${ }^{5}$ Geschichte der Hohenstaufen und ihrer Zeit, 6 Bde, 1823-25 bei Brockhaus in Leipzig veröffentlicht.

${ }^{6}$ Das Historische Taschenbuch gab Raumer zwischen 1830 und 1867 heraus.

${ }^{7}$ Die Antiquarischen Briefe, 1851 bei Brockhaus in Leipzig erschienen, zeugen von seinem Dialog mit seinem Kollegen August Boeckh (s. dazu auch eine Bemerkung aus dem Brief von George Grote, S. 59 des Korrespondenzkastens des Nachlasses Raumer aus der Staatsbibliothek zu Berlin - Preußischer Kulturbesitz). ${ }^{8}$ Die beiden Erzählungen Wilhelmine (im Lesegarten 1855 erschienen) und Marie (im Literarischen Nachlass 1869 erschienen).

${ }^{9}$ So reiste er 1816 durch die Schweiz und Italien, 1830 nach Frankreich, 1835 nach England, 1839 nach Italien, 1843 in die USA. Zur Schilderung seiner späteren Reisen, s. Literarischer Nachlass (wie Anm. 1), S. 3.

${ }^{10}$ Dabei geht es um einzelne Briefe an ihn, die im Nachlass Raumer der Staatsbibliothek zu Berlin -

Preußischer Kulturbesitz zu finden sind. Weitere ungedruckte Briefe befinden sich sowohl in Krakau in der Jagellionischen Bibliothek als auch im Geheimen Staatsarchiv (Berlin).

${ }^{11}$ S. W. Friedrich (wie Anm. 1), S. 15: „Hardenberg, der eben zum Staatskanzler ernannt worden war, rief ihn an seine Seite, ja, er nahm ihn in sein Haus auf. So kam es, daß er nunmehr einen hervorragenden Anteil an dem preußischen Reformwerk nahm. Der Volksmund nannte ihn den ,kleinen Staatskanzler“.“ “
} 
Maaßnehmungen, welche einen lange überlegten und wohlgeordneten Entschluß zu Gunsten der Wissenschaft deutlich aussprachen. ${ }^{12}$

Der Übergang zwischen politischer und akademischer Karriere erfolgte in der Tat nicht in Form eines eklatanten Bruches: Weder kam es zu gravierenden Zwistigkeiten mit der Regierung, ${ }^{13}$ noch gab Raumer seine politischen Ansprüche von einem Tag auf den anderen auf. Besonders an der Breslauer Universität, wo sowohl unter den Studenten als auch unter dem akademischen Personal das politische Bewusstsein besonders ausgeprägt war, fand er Raum zur Verbreitung selbstbewusster politischer Stellungnahmen, die er zum Teil auch in der Öffentlichkeit vertrat. ${ }^{14}$

Ganz unproblematisch war das für einen Universitätsprofessor natürlich nicht. So erfahren wir durch einen unveröffentlichten Brief seines Jugendfreundes Karl Wilhelm Ferdinand Solgers ${ }^{15}$ vom 26. April 1812, dass Raumer von diesem nur mit äußerstem Nachdruck davon abgehalten werden konnte, einen wütendes Pamphlet gegen Fichte zu veröffentlichen. Solgers Argumente zeigen die Schwierigkeiten Raumers auf, sich in sein neues Amt als Professor einzufügen. Ehe näher darauf eingegangen wird, sei der Kontext kurz geschildert, in dem der Brief entstand.

Infolge von mehreren Disziplinproblemen an der Berliner Universität kam es zu Beginn des Jahres $1812 \mathrm{zu}$ starken Uneinigkeiten zwischen dem Rektor, Fichte, und dem Senat derselben. Indem er sich schließlich direkt an das Ministerium wandte, ohne den Senat zu konsultieren, verursachte Fichte eine regelrechte Institutionskrise innerhalb der ohnehin noch nicht sehr stabilen Universität. ${ }^{16}$ Dadurch zog er sich den Unmut beinahe aller Professoren zu, selbst derjenigen, die ihm, wie etwa Solger, sein einziger Kollege im philosophischen Fach, im Vorfeld nicht unbedingt abgeneigt waren. Auf einen besonders vehementen Brief Solgers (vom 22. März 1812) ${ }^{17}$ reagierte Raumer mit der Verfassung einer Fichte-Kritik, die er zu drucken beabsichtigte. Doch ehe er den Text in den Druck gab, schickte er ihn an Solger. Zügig antwortete ihm dieser auf die Sendung. Solger, aus dessen Brief sich Raumers Wutanfall genährt hatte, konnte dem Inhalt der Kritik nur zustimmen. ${ }^{18}$ Dennoch machte Solger, der über etwas mehr Erfahrung im akademischen Betrieb verfügte, seinem Freund deutlich, dass nicht jede Wahrheit eine - sei es explizit oder anonym - mit dem Namen eines Professoren versehene Veröffentlichung wert ist. Sein erstes Argument betrifft die bereits prekäre Balance in der kleinen akademischen Welt Preußens, die es zu schonen gelte:

\footnotetext{
${ }^{12}$ Schleiermacher, Literarischer Nachlaß, Zur Philosophie, Berlin 1835, Bd. 1, S. 174.

${ }^{13}$ S. Friedrich (wie Anm. 1), S. 16.

${ }^{14}$ S. Lebenserinnerungen (wie Anm. 1), Bd. 1, S. 250.

${ }^{15}$ Karl Wilhelm Ferdinand Solger (1780-1819), Philosoph und Philologe, studierte zu gleicher Zeit wie Friedrich von Raumer in Halle Jura und klassische Philologie. Beide traten der sog. Freitag-Gesellschaft bei. Dieser Freundeskreis wurde zum Zeitpunkt des Hallenser Studiums gegründet und versammelte sich bis in die Mitte des 19. Jahrhunderts, s. Lebenserinnerungen (wie Anm. 1), Bd. 2, S. 106. 1809 bekam Solger einen Ruf nach Frankfurt/Oder. 1811 wurde er dann mihtilfe von Raumers Unterstützung nach Berlin berufen, wo er bis zu seinem plötzlichen Tod 1819 an der Universität Philologie und Philosophie lehrte.

${ }^{16}$ Dazu, s. Max Lenz, Geschichte der Königlichen Friedrich-Wilhelms-Universität zu Berlin, Halle 1910, Bd. 1, S. 412-430.

${ }^{17}$ Dieser Brief ist abgedruckt und kommentiert in: Anne Baillot, „Wie rehabilitiert man einen Schriftsteller und wozu? Das Beispiel unerschlossener Briefwechsel aus dem Umkreis des Dichters Ludwig Tieck, des Philosophen Karl Solger und des Historikers Friedrich von Raumer“ in Dokument/Monument. Textvarianz in den verschiedenen Disziplinen der europäischen Germanistik - Akten des 38. Kongresses des französischen Hochschulgermanistikverbandes, hg. v. F. Lartillot und A. Gellhaus, Bern 2008, S. 103-126.

18 S. Brief Solgers an Raumer vom 26. April 1812, NL Raumer, Korrespondenzkasten, S. 130 sqq.
} 
[so] sieht es aus, wie ein Antagonismus der Universitäten Breslau und Berlin, und daß sich ein solcher nicht bilde, besonders auf eine leidenschaftliche Art, muß gewiß möglichst vermieden werden.

Auch auf der Ebene der Beziehungen unter Professoren legt Solger auf die Vermittlung eines positiven Bildes gegenüber der breiteren Öffentlichkeit Wert:

Lassen Sie uns ja beitragen, eine würdige Begegnung unter Gelehrten aufrecht zu halten, und auch die Würde der Universitäten selbst und ihrer Häupter zu schützen, die jetzt so vielem Abbruch ausgesetzt ist. Einen Rector einer fremden Universität sollte man fast wie einen fremden Hof behandeln.

Solger wird wohl dabei die Vervielfachung der Gelehrtenstreitigkeiten seit dem Ende des 18. Jahrhunderts und der damit zusammenhängenden Veröffentlichungstätigkeit im Blick gehabt haben, wodurch sich der eine oder andere in der Tat stark diskreditierte. Doch an seinem letzten Satz lässt sich ablesen, dass er seinem Freund vermitteln möchte, dass dieser in seiner neuen Funktion noch einiges dazu zu lernen hätte. Sein letzter und „wichtigster“ Grund bringt dies explizit zum Ausdruck:

Legen Sie ja nicht einen zu großen Werth auf den Beifall, den Ihnen die Studenten bezeigt haben. [...] Man verlangt nachher, daß Sie einen solchen Ton halten sollen, was gewiß nicht Ihre Sache sein wird. Ganz anders wird es den Studenten vorkommen, wenn Sie nun mit Strenge und Ernst Ihre Wissenschaften vortragen, ohne zu ihrer Trägheit herabzulassen, und ihnen vorzukauen.[...] Nur der Beifall ist hier eigentlich werth, der früchtet, und dieser wird nur sehr nach und nach, und durch treues, sorgfältiges Lehren erworben. [...] Sehn Sie auf Bredows Beispiel. ${ }^{19}$ Dieser hatte in Frankfurt einen großen Zulauf und Beifall, und hat sich diesen größtentheils durch gewisse Tiraden und Abschweifungen verschafft, welche die Leidenschaft der jungen Leute erregten. Die wahre und einzige Leidenschaft aber, die wir erregen sollen, ist die für Wahrheit und das Rechte, und die kann nur die Wissenschaft selbst und ihre reine Darstellung hervorbringen.

In letzterem Punkt werden wohl Solger und Raumer bei aller Freundschaft nie einer Ansicht gewesen sein: Denn für Raumer war eine Trennung von Wissenschaft und Politik schlicht unmöglich. Und auch wenn seine Gegenbriefe nicht erhalten sind und seine Antwort auf Solgers Ermahnungen nicht ermittelt werden kann, beweisen der Ton und die Geschwindigkeit von Solgers Reaktion, dass zumindest in diesem Fall Raumer eine Grenze überschritten hatte. Dies ist der einzige, mir bekannte Fall einer so gravierenden Uneinigkeit zwischen beiden Männern im Laufe ihrer lebenslangen Freundschaft. An der Entwicklung von Universitätsstatuten, welche Alleingänge wie diejenigen Fichtes vermeiden sollten, arbeiteten beide Männer für die Universitäten von Berlin bzw. Breslau in den Folgejahren

${ }^{19}$ Gottfried Gabriel Bredow (1773-1814), klassischer Philologe und überzeugter Patriot. 
gemeinsam. ${ }^{20}$ Und nicht zuletzt um Solger geographisch näher zu sein, ${ }^{21}$ freute sich Raumer über seine Berufung nach Berlin 1819. ${ }^{22}$

Leider verstarb der noch junge Philosoph ausgerechnet zum Zeitpunkt, an dem Raumer in Berlin eintraf. $\mathrm{Ob}$ seine Berliner Karriere eine andere gewesen wäre, wenn ihm Solger beigestanden hätte? ${ }^{23}$ Sicherlich hätte Solger bei weiteren Reden und Schriften Raumers provokante Passagen streichen wollen. Von solchen Äußerungen und ihren Folgen möchte ich nun zwei Beispiele geben: das erste im Kontext des Oberzensurkollegiums, das zweite im Kontext der Akademie der Wissenschaften.

\section{Zensor wider Willen}

Das Oberzensurkollegium ${ }^{24}$ wurde 1819 in Folge der Karlsbader Beschlüsse gegründet. Obwohl es ursprünglich zum Schutz der Pressefreiheit konzipiert war, ${ }^{25}$ entwickelte es sich rasch zu einem expliziten Zensurorgan, ${ }^{26}$ das zusätzlich $\mathrm{zu}$ den bereits vorhandenen Zensureinrichtungen das Ziel hatte, die politische Presse zu zügeln, seine Aufmerksamkeit aber auch gelegentlich Büchern widmete. ${ }^{27}$ Ohne klar definierte Aufgaben, vor allen Dingen ohne Mittel und den drei Ministern des Innern, der geistlichen und der äußeren Angelegenheiten unterworfen, ${ }^{28}$ hatte das Oberzensurkollegium nur einen begrenzten Spielraum. So erlahmte auch dessen Aktivität nach wenigen Jahren. Der erste Vorsitzender war Raumers Onkel, der Wirkliche Geheime Legationsrat Karl Georg von Raumer, neben ihm wurden im Gründungsjahr elf Mitglieder bestimmt. ${ }^{29}$

Obwohl er sich es nicht gewünscht hatte, gehörte Friedrich von Raumer zu diesen elf. ${ }^{30}$ Dass ein ausgeprägtes Zensurwesen prinzipiell seinen eigenen Überzeugungen nicht entsprach, lässt sich seinem Kündigungsschreiben aus dem Jahr 1831 entnehmen. Auslöser seines Abgangs war die Verstümmelung der Druckfassung seiner Festrede als Rektor der Universität 1822 - durch das Oberzensurkollegium selbst. ${ }^{31}$ Das Verfahren war für Raumer umso ärgerlicher, als der König selbst die Rede gebilligt hatte. Über diesen Zwischenfall

\footnotetext{
${ }^{20}$ S. zB Briefe von Solger an Raumer vom 28.01.1814 (z:T. in Solgers Nachgelassenen Schriften und Briefwechsel, Leipzig 1826, S. 607-614), NL Raumer, Korrespondenzkasten, S. 169 sqq und vom 6. Dezember 1818, NL Raumer, Korrespondenzkasten, S. 176 sqq.

${ }^{21}$ Was 1811 misslungen war: s. Lebenserinnerungen, Bd. 1, S. 241.

22 S. Lebenserinnerungen (wie Anm. 1), Bd. 1, S. 258. Zu Solgers Anteil an Raumers Berufung nach Berlin, S. Brief Solgers an Raumer vom 6.12.1818 (wie Anm. 20).

${ }^{23}$ Schleiermacher kommt in seiner Rede zur Aufnahme Raumers an der Akademie relativ detailliert auf Solgers Laufbahn zu sprechen. Anlass dieser Erwähnung ist für Schleiermacher die damals noch frische Herausgabe von Solgers Nachgelassenen Schriften und Briefwechsel durch Ludwig Tieck und Friedrich von Raumer, die von der Intensität dieser Freundschaft zeugt.

${ }^{24}$ Zum Oberzensurkollegium, s. Friedrich Kapp, „Die preußische Preßgesetzgebung unter Friedrich Wilhelm III (1815-1840). Nach den Akten im Königl. Preußischen Geh. Staatsarchiv“, in: Archiv für Geschichte des Deutschen Buchhandels 6 (1881).

${ }^{25}$ Insbesondere solange Hagemeister lebte, s. Kapp (wie Anm. 24), S. 195-197.

${ }^{26}$ S. Kapp (wie Anm. 24), S. 205.

${ }^{27}$ S. die Bemerkungen Friedrich Kapps (wie Anm. 24) zu der an Tiecks Novellen durchgeführten Zensur 1822 , S. 220 .

${ }^{28}$ S. F. Kapp (wie Anm. 24), S. 206

${ }^{29}$ S. Kapp (wie Anm. 24), S. 205-206. Doch fleißig waren bei weitem nicht alle, und Personalwechsel kamen relativ oft vor; s. Kapp, S. 225-228.

${ }^{30}$ Friedrich Kapp (wie Anm. 24) unterstreicht, dass Raumer in den ersten Jahren seine Pflichten als Mitglied des OZK wahrnahm (S. 229).

${ }^{31} \mathrm{Zu}$ diesem Vorfall, s. Lebenserinnerungen (wie Anm. 1), S. 99-117 und Kapp (wie Anm. 24), S. 229-232.
} 
hinaus kritisierte Raumer in seinem Kündigungsschreiben an seinen Onkel den Sinn der Einrichtung:

Anstatt nämlich die schreibende und lesende Welt für größere, echte Freiheit zu erziehen und, ich möchte sagen der literarischen Großjährigkeit immer näher zu bringen, hat vielmehr die Strenge und Aengstlichkeit der Aufsicht allmählich zugenommen, sodaß Preußen (einst in dieser Beziehung der freigesinnteste und der Treue, sowie dem Verstande seiner Untertanen am meisten vertrauende Staat) jetzt fast hinter allen anderen zurücksteht.

Die Zahl der Verbote von Büchern und Zeitschriften wächst, obgleich dieser geistige Cordon das etwaige Böse noch weniger abhalten oder vernichten kann, als der jetzt aufgegebene, medicinisch-militärische bei Cholera. ${ }^{32}$

Dieses seinem Onkel als Leiter des Oberzensurkollegiums bestimmte Schreiben wurde ohne Raumers Wissen und Zustimmung in der Presse bekanntgemacht. ${ }^{33}$ Damit zog er sich den Argwohn des Königs zu, der ihm am 21. Juli 1833 schrieb:

Ich habe keine Bedenken gefunden, Sie nach Ihrem Wunsche als Mitglied des OberCensur-Kollegiums zu entlassen und Sie fernerhin von den Arbeiten desselben um so mehr zu entbinden, als Sie sich ihnen früher schon auf eine ungehörige Weise entzogen haben, kann jedoch nicht umhin, Ihnen meine ernstliche Mißbilligung der verunglimpfenden Fassung Ihres Entlassungsgesuches vom 30. Oktober 1831 und dessen Verbreitung durch Mittheilung an andere Personen, die den Mißbrauch einer öffentlichen Bekanntmachung zur Folge gehabt hat, zu erkennen zu geben [...]. Ich erwarte, daß Sie zu solchen Vorwürfen fernerhin keine Veranlassung mehr geben werden. ${ }^{34}$

Dennoch drehte sich der Vorfall zu Raumers Gunst - nämlich in liberaleren Kreisen sowie im Ausland. So liest man in einem Brief vom französischen Publizisten Saint-Marc-Girardin:

Ce ne sont pas seulement vos livres, Monsieur, qu'il faut admirer; ce sont vos actions. Votre demission des fonctions de censeur vous fait en France un renom populaire. Vous etes plus heureux que beaucoup de nos hommes d'etat. Vous reunissez la popularite et la gloire. Dans notre pays elles sont trop souvent separees l'une de l'autre. ${ }^{35}$

Hiermit wird auf eine mehrspurige Rezeption hingewiesen. Zuerst eine innenpolitische, preußische, in der Raumer eine Persönlichkeit in einer Konstellation zahlreicher Staatsdiener ist, die entweder in der Politik und Verwaltung oder in einer akademischen Institution versuchten, sich einen Weg zu Machtpositionen zu bahnen. Darüber hinaus aber verfügte Friedrich von Raumer über ein Prestige, das auf seinen Schriften und Taten beruhte und ihn

\footnotetext{
${ }^{32}$ LE, Bd. 2, S. 357. Kapp erklärt, dass K. von Raumer sich ursprünglich verweigerte, das Schreiben den Ministern zu zeigen, sein Neffe ihn aber dazu zwang (wie Anm. 24, S. 230-231.

${ }^{33}$ Raumer fügte seine Verteidigungsakte den Lebenserinnerungen bei (Bd. 2, . 356-360). Daraus wird klar, dass Brockhaus Gehilfe, Schönrock, seinem ehemaligen Meister, Erhart, in Stuttgart eine Kopie des Schreibens hat zukommen lassen. „Letzerer aber druckte sie in der von ihm verlegten Suttgarter Allgemeinen Zeitung ab, von wo das Schriftstück zunächst seinen Weg in die Allgemeine Augsburger, Speyerer, Casseler, Bremer Zeitung und den Nürnberger Korrespondenten fand. Raumer möchte sich entschuldigen, soviel er wollte, und den Sachverhalt erklären, es half nichts, und er wurde sogar polizeilich vernommen." (Kapp, wie Anm. 24. S. 231). ${ }^{34}$ Zitiert von Kapp (wie Anm. 24), S. 232-234.

${ }^{35}$ NL Raumer, Korrespondenzkasten, Brief von Saint-Marc-Girardin, S. 105.
} 
zum intellektuellen Weltbürger machte. So verhalf ihm seine Politik nach außen zu einer etablierten Funktion als Mittler in einem weltweiten Netzwerk, wovon seine erhaltene AnKorrespondenz zeugt. Dies lässt sich am Beispiel seiner Tätigkeit an der Akademie der Wissenschaften deutlicher noch belegen.

\section{Vom Akademie-Mitglied zum „Marquis Posa“}

Neben seiner langjährigen Tätigkeit an der Berliner Universität, wo er zu der ersten Generation der Historiker vom Fach angehörte, wurde Friedrich von Raumer 1827 Akademie-Mitglied und bis 1847 ständiger Sekretär der historisch-philologischen Klasse. In seiner bereits erwähnten Rede zu Raumers Aufnahme an der Akademie hebt Friedrich Schleiermacher die intellektuellen Grundzüge des neuen Mitgliedes hervor:

Sie, mein Herr, haben schon während Sie noch Staatsmann waren als politischer Schriftsteller in Ihren Dialogen Ueber Krieg und Handel, ${ }^{36}$ in Ihrem brittischen Besteuerungssystem, ${ }^{37}$ gezeigt, wie derjenige die Gegenwart betrachtet, welchem die Entwikklung der menschlichen Dinge in ihren allgemeinen Grundzügen wie in den eigenthümlichen Formen verschiedener Zeiten und Zonen lebendig vorschwebt. Seitdem haben Sie sich keinem von den verschiedenen Zweigen, welche diese Klasse der Akademie in sich vereinigt, fremd gezeigt. ${ }^{38}$

Mit dem Begriff des ,,politischen Schriftstellers“ trifft Schleiermacher genau, was Raumers Wirkungssphäre definiert. ${ }^{39}$ Eine enge Verbindung von akademischer Statur und politischem Denken wird ebenfalls in François Pierre Guillaume Guizots Dankschreiben an Raumer zu seiner Ernennung zum auswärtigen Mitglied der Berliner Akademie der Wissenschaften ermittelt. ${ }^{40}$ In dieser Angelegenheit hatte Raumer als Sekretär der historisch-philologischen Klasse den französischen Staatsmann angeschrieben. Bezeichnenderweise knüpft Guizot an eine Passage in Raumers Brief an, die weniger mit der akademischen Tätigkeit als mit der politischen Praxis zu tun hat:

Je ne vous remercie pas moins, Monsieur, des vœux que vous m'exprimez pour le succès de la politique de paix et de bonne intelligence Européenne que je m'efforce de faire prévaloir de nos jours, dans l'état actuel de la civilisation et de l'esprit humain, la

\footnotetext{
${ }^{36}$ Sechs Dialoge über Krieg und Handel, 1806 in Berlin unter Herausgabe von Johannes von Müller erschienen. ${ }^{37}$ Das Brittische Besteuerungs-System: insbesondere die Einkommensteuer, dargestellt, mit Hinsicht auf die in der Preussischen Monarchie zu treffenden Einrichtungen, Berlin 1810.

${ }^{38}$ Schleiermacher, Rede zur Aufnahme Raumers an der Akademie der Wissenschaften, 3. Juli 1827.

${ }^{39}$ Die Rezeption von Raumers Veröffentlichungstätigkeit als Produkt eines Intellektuellen mit Anspruch auf Allgemeingültigkeit ging sogar über die Grenzen Preußens hinaus. So schrieb ihm der französische Publizist Saint-Marc Girardin - auch hier als Lob (bzw. Selbstlob!) zu verstehen: „Votre developpement historique de l'idee de droit, de l'etat et de la politique est sous la forme d'articles separés que vous lui avez laissée une histoire complete de la science politique ou de la civilisation. Tel est l'avantage de tout ce qu'écrivent les hommes de notre trempe, Monsieur. Ils generalisent tout. dans les plus petits details, ils embrassent beaucoup. Comme les grands peintres de paysage et de marine, ils enferment dans un cadre etroit une grande et admirable perspective.“, in : NL Raumer, Korrespondenzkasten, Brief von Saint-Marc-Girardin an Raumer, undatiert, S. 104-105.
}

${ }^{40}$ S. Brief von Guizot an Raumer vom 12. Dezember 1840, NL Raumer, Staatsbibliothek zu Berlin - PK, Korrespondenzkasten, S. 60: „J'ai été vivement touché de l'honneur que vient de me faire l'Académie des Sciences de Berlin en me conférant le titre de Membre étranger.“ 
guerre sans nécessité absolue et sans justice évidente seroit une monstruosité. Croyez, Monsieur, que cela est compris et senti en France aussi bien qu'en Allemagne ; et ne laissez pas reparoître parmi vous des préventions, des minorités nationales qui rendroient la tâche des amis de la paix bien plus difficile et leur succès plus incertain. ${ }^{41}$

Auch ohne Kenntnis von Raumers Originalbrief, zeigt sich an diesem Schreiben, wie er seine höchsten akademischen Ämter mit einer politisch-diplomatischen Funktion verband und unter diesem doppelten Gesicht in der Weltpolitik hervortrat.

Doch auch hier hatte die Politisierung des akademischen Diskurses ihre Grenzen. Diese überschritt Raumer mit der Rede auf Friedrich II. von Januar 1847, die er als ständiger Sekretär der historisch-philologischen Klasse zu halten hatte. In dieser Rede äußerte sich Raumer Friedrichs Toleranzedikt in Anwesenheit des Thronfolgers. ${ }^{42}$ Der Historiograph der Raumer-Familie hebt bei der Schilderung des anschließenden Vorfalls, beinahe 150 Jahre später, die gleichen Einwände heraus, die Solger seinem Freund 1812 gemacht hatte:

Daß der Redner nun auch seinerseits scharf wurde, wird ihm niemand verargen; aber der Ton seiner Polemik war nicht der akademische [...]. Viel bedenklicher aber war es, daß er auch in der Sache die Grenze nicht einhielt und sich nach einer ausgezeichneten Vertheidigung jenes friedericianischen Worts in Ausführungen erging, die im Sinne einer Warnung für die Monarchen, der die Rede anhörte, verstanden werden mußte. ${ }^{43}$

In der Vormärzstimmung, die in der preußischen Hauptstadt herrschte, bewirkten die Rede und der darauffolgende Abgang Raumers aus der Akademie ${ }^{44}$ jedoch alles andere als eine Verurteilung in der öffentlichen Meinung. Im Gegenteil: Seine Geste verhalf ihm in Preußen und Europa als neuer „Marquis Posa“ zum Ruhm. ${ }^{45}$ Kurz darauf wird er Mitglied der Frankfurter Nationalversammlung. Dennoch war damit seine Rückkehr in die Politik nicht endgültig vollzogen. Er erwies sich dort als erstaunlich wirkungslos und gab nach der ersten Legislaturperiode diesen Versuch auf. ${ }^{46}$

${ }^{41}$ Brief von Guizot an Raumer vom 12. Dezember 1840, NL Raumer, Staatsbibliothek zu Berlin - PK, Korrespondenzkasten, S. 60v.

${ }^{42}$ S. W. Friedrich (wie Anm. 1), S. 37.

${ }^{43}$ H. v. Raumer (wie Anm.1 ), S. 107-108.

44 Zunächst beabsichtigte er nur, als Sekretär abzutreten; nachdem der Entschuldigungsbrief der Akademie aber in die Presse kam, tritt er komplett von der Akademie ab.

${ }^{45}$ S. Werner (wie Anm. 1), S. 37: „Die Angelegenheit bekam dadurch noch ein unangenehmes Nachspiel, daß der Minister Eichhorn es für nötig erachtete, das Schreiben der Akademie in der Presse zu veröffentlichen. Das nur für den König bestimmte, fast servile Entschuldigungsschreiben brachte der Akademie in der Öffentlichkeit Spott und Haß ein. Raumer [...], der ,allein Männerstolz vor Königsthronen bewährt“ hatte, erreicht jetzt den Höhepunkt seiner Popularität. Überall wurde er als Marquis Posa gefeiert, in den Zeitungen fand die Angelegenheit stärksten Widerhall“

${ }^{46}$ Er berichtet selber in seinem Literarischen Nachlaß (wie Anm. 1) nüchtern darüber, s. ebd. Bd. 1, S.1: „Mein Austritt aus der berliner Akademie (1847, worüber ich vor der Hand noch schweige) machte mich, gegen die Erwartung Vieler, so bekannt und beliebt, daß ich zum Stadtverordneten erwählt wurde. Ich habe die mir dadurch auferlegten Pflichten mit freudiger und eifriger Theilnahme zu erfüllen gesucht [...]. Ueber meinen Aufenthalt und meine, meist fruchtlose, Thätigkeit als Reichstagsabgeordneter in Frankfurt am Main und in Paris, geben meine gedruckten Briefe umständliche Auskunft.“; sowie schlussstrichziehend auf S. 3 ebd.: „Hiermit nahm meine politische Thätigkeit ein Ende, und ich hatte Gründe genug eine Erneuerung nicht zu wünschen. Ich habe durch dieselbe viel gelernt, aber keinen bekehrt, und nichts bewirkt". Seinen geringen Einfluss begründet Raumer u.a. damit, dass er sich keiner Partei anschloss. 
Nach dieser zweiten, erfolglosen politischen Erfahrung ging Raumer erneut auf Reisen. ${ }^{47}$ Denn als Vertreter Preußens im Ausland war er in der Tat alles andere als wirkungslos. Strategische Gesten verhalfen ihm dazu: so ließ er etwa ausgewählten ausländischen Politikern und Gelehrten seine Werke zukommen. Einige kannte er von seinen Reisen, ${ }^{48}$ mit anderen verband ihn eine akademische Beziehung. ${ }^{49}$ Auf diese Weise wurde er international wahrgenommen. Dem Nachlass lassen sich überzeugende Belege dafür aus den USA, England und vor allem Frankreich ${ }^{50}$ hinzufügen. Mit dem ihm üblichen Schwung nahm der französische Publizist Saint-Marc-Girardin zu Raumers Untergang Polens wie folgt Stellung:

C'est rendre service a la France que de lui faire connaitre des ouvrages aussi remarquables que les sont les votres. ${ }^{51}$

Seine englischen und nord-amerikanischen Briefpartner ihrerseits wiesen noch expliziter auf den Nutzen hin, den sie von Raumers Schriften haben. So schrieb ihm der amerikanische Historiker William Hickling Prescott im April 1846:

It has been a pleasant thing to your friends on this side of the water to read the fruits of your travels and scientific investigations on the valuable work which has come from your pen. ${ }^{52}$ It is impossible to read the speculation of an enlightened foreigner on our domestic institutions, without getting valuable hints, the more valuable as not likely to present themselves to the mind of a native, familiarized from the cradle to the operation of these institutions. [...] All the intelligent of my countrymen, whom I have conversed with on the subject, accord high merit to your work on the score of extensive information \& very sagacious reflection, even where they differ from you as to the conclusions. ${ }^{53}$

In dieser Konstellation wusste sich Raumer optimal zu positionieren. Der vermeintliche Mangel an Tiefe, die lose Struktur, die fehlerhafte Wissenschaftlichkeit, die ihm im deutschsprachigen Raum vorgeworfen wurden, ${ }^{54}$ werden international ganz anders wahrgenommen. Hier wird ihm nicht nur eine gute Kenntnis der Sachlage zugebilligt, sondern darüber hinaus noch seinen Stil gelobt. ${ }^{55}$ So schreibt Prescott, besonders angenehm sei seine ,calm and dispassionate“ Manier. ${ }^{56}$

Trotz seiner im Wesentlichen viel kritischeren Einstellung als der Prescotts lässt sich der Brief des Grafen von Stanhope vom 10. August 1841 im Ansatz ähnlich interpretieren. Stanhope inszeniert sich und Raumer als die jeweiligen Vertreter von zwei Nationen, zwei Interessen und daher zwei politischen Einstellungen, die zwar auseinandergehen, aber im

\footnotetext{
${ }^{47}$ S. Literarischer Nachlaß (wie Anm. 1), S. 3.

${ }^{48}$ S. Literarischer Nachlaß (wie Anm. 1), S. 3.

${ }^{49}$ S. Brief vom Institut de France vom 20. Okt. 1866, NL Raumer, Korrespondenzkasten, S. 102.

${ }^{50}$ Zur Intensität der französischen Raumer-Rezeption, s. u.a. Literarischer Nachlaß (wie Anm. 1), Bd.1, S. 3,

Liste der französischen Ansprechspartner (vgl. mit Liste der Engländer, Amerikaner, etc., auf S. 4).

${ }^{51}$ Brief von Saint-Marc-Girardin, NL Raumer, Korrespondenzkasten, S. 104.

${ }^{52}$ Die Vereinigten Staaten von Amerika, 1845.

${ }^{53}$ Brief von William Hickling Prescott vom 30. April 1846, NL Raumer, Korrespondenzkasten, S. 82.

${ }^{54}$ S. H. v. Raumer (wie Anm. 1), S. 104-105.

55 Ähnliches lässt sich den Briefen des englischen Historikers George Grotes aus dem handschriftlichen Nachlass entnehmen.

${ }^{56}$ Brief von William Hickling Prescott vom 30. April 1846, NL Raumer, Korrespondenzkasten, S. 83.
} 
Gespräch mit einander bleiben. ${ }^{57}$ Dennoch ist es nicht Stanhope, sondern Prescott, der Raumer explizit zum humanistischen Weltbürger krönt:

The broad and generous views which you take of all the great features of our public policy commend themselves to every true lover not only of this country, but of the human race. For your views are those of a citizen of the world. ${ }^{58}$

Dieses Idealbild Raumers als Weltbürger weist an erster Stelle auf seine Mittlerfunktion hin, die in der Tat aus ihm in der Gelehrtenrepublik einen preußischen Diplomat ersten Ranges machte. Dass sein langes Leben zu dieser Stellung beigetragen hatte, wusste er selbst. 1861 eröffnete er seine Lebenserinnerungen und Briefwechsel mit einer Selbststilisierung, welche Politisches, Akademisches und Persönliches aufs Engste verbunden inszeniert:

1861.

Den 14. Mai bin ich volle 80 Jahre alt.

Den 9. September bin ich 50 Jahre Professor.

Den 5. October bin ich 50 Jahre Doctor.

Den 20. Oktober bin ich 50 Jahre verheiratet.

Den 8. December bin ich 60 Jahre in königlichem Dienste.

Den 20. Dezember bin ich 60 Jahre Mitglied der Singakademie. ${ }^{59}$

Eben dieses Bild eines wackeren, beinah unzerstörbaren Intellektuellenlebens fasste sein Briefpartner George Grote zusammen, indem er ihn einen „Isokrates“600 nannte. Auch wenn Raumers Bedeutung der des Sokrates nicht standhält - sicher ist, dass seine Schriften und seine Taten die Komplexität der Beziehungen zwischen Politik und Wissenschaft im Berlin des 19. Jahrhunderts besser auffassen helfen.

\footnotetext{
57 „Es ist mir nicht möglich Ihre Ansichten zu theilen; indeß erkenne ich gern mit wie viel Aufrichtigkeit und Treusinn diese Ansichten von Ihnen behaubtet sind. Ganz unpartheiisch sind wir vielleicht beide nicht.- Es ist möglich daß mir das Wohlseyn meines Vater's Pächter und mein eigenes GeldSackes etwas zu nahe am Herzen liegt; an Ihrer Seite haben Sie vielleicht - als ein guter Preusse und Patriot - nicht vergessen wie viel der LandBauer in Posen und der Handelsmann in Dantzig an einen offenen Korn-Handel mit England zu gewinnen hätten.“: Brief von Philip Henry Earl of Stanhope vom 10. August 1841, NL Raumer, Korrespondenzkasten, S. 190.

${ }^{58}$ Brief von William Hickling Prescott vom 30. April 1846, NL Raumer, Korrespondenzkasten, S.82-83.

${ }^{59} \mathrm{~S}$. Lebenserinnerungen (wie Anm. 1), unnummerierte Frontseite.

${ }^{60}$ S. Brief von George Grote an Raumer vom 11. Februar 1862, NL Raumer, Korrespondenzkasten, S. 56: „It is interesting for me to learn from your preface that you have already passed your eightieth year. I trust that your career of literary activity, long as it has already been, may be yet farther prolonged, together with such reasonable measure of bodily health as is indispensable to the exertion of mental power. Isokrates was 82 years

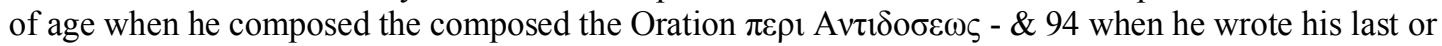
Panathenaic Oration -“"
} 